J. Lake Sci.(湖泊科学), 2008, 20(1): 51-57

http://www.jlakes.org. E-mail: jlakes@niglas.ac.cn

(C)2008 by Journal of Lake Sciencesa

\title{
表面活性剂在滇池水体中的生物降解*
}

\author{
王 智 ${ }^{1,2}$, 吴幸强 ${ }^{1,2}$, 肖邦定 ${ }^{1 * *}$, 涂新海 ${ }^{1,2}$ \\ (1: 中国科学院水生生物研究所, 武汉 430072) \\ (2: 中国科学院研究生院, 北京 100049)
}

摘 要: 利用河水衰减法研究阴离子表面活性剂(LAS)和非离子表面活性剂(NIS)在滇池水体中不同条件下的生物降解规律. 结果表明: 在滇池自然水体中, LAS 和 NIS 均能生物降解，在试验时间(30d)内, LAS 的降解率达 $95 \%$ 以上, NIS 的降解率达 $92 \%$ 以上, 其降解动力学遵从二级动力模型. 改变水温、表面活性剂初始浓度、 $\mathrm{pH}$ 值以及添加营养物质(葡萄糖或磷酸氢二钠)均 对 LAS 和 NIS 的降解有一定的影响. 水温对表面活性剂生物降解影响最大, 当水温从 $20^{\circ} \mathrm{C}$ 增至 $30^{\circ} \mathrm{C}$ 时, LAS 的降解速率从 $0.73 \mathrm{~d}^{-1}$ 增至 $2.10 \mathrm{~d}^{-1}$, NIS 降解半衰期从 $11.5 \mathrm{~d}$ 减至 $4.4 \mathrm{~d}$; 表面活性剂初始浓度增加, 降解半衰期有所增加; 表面活性剂在 $\mathrm{pH} 7$ 时的降解性能略优于 $\mathrm{pH} 10$ 时的降解性能; 添加葡萄糖抑制 LAS 和 NIS 的降解，而添加磷源磷酸氢二钠对其降解有一定的促 进作用; 曝气能促进 LAS 的降解, 但对 NIS 降解的促进作用并不明显.

关键词: LAS; NIS; 生物降解; 降解动力学; 滇池

\section{Biodegradation of surfactants activators in water of Lake Dianchi}

\author{
WANG Zhi ${ }^{1,2}$, WU Xingqiang ${ }^{1,2}$, XIAO Bangding ${ }^{1} \&$ TU Xinhai $^{1,2}$ \\ (1: Institute of Hydrobiology, Chinese Academy of Sciences, Wuhan 430072, P.R. China) \\ (2: Graduate School of Chinese Academy of Sciences, Beijing 100049, P.R. China)
}

Abstract: Biodegradation of anion-surfactant (LAS) and non-ion surfactant (NIS) in water of Lake Dianchi under the different incubation conditions were studied by the 'river die-away' method in this paper. The results showed that LAS and NIS could be biodegradated by microorganisms in the water of Lake Dianchi. The percentages of degradation of LAS and NIS were more than $95 \%$ and $92 \%$ after 30 days respectively. The biodegradation of LAS and NIS fitted the second kinetic model and the correlation coefficient values were among 0.956 to 0.999 . Incubation temperature, initial concentrations of surfactants, $\mathrm{pH}$ value and nutrients (glucose or $\mathrm{Na}_{2} \mathrm{HPO}_{4}$ ) could affect the biodegradation of LAS and NIS, Incubation temperature was the major factor influence on biodegradation of surfactant in water of Lake Dianchi. When the incubation temperature increased from $20^{\circ} \mathrm{C}$ to $30^{\circ} \mathrm{C}$, the biodegradation rate of LAS increased from $0.73 \mathrm{~d}^{-1}$ to 2.10 $\mathrm{d}^{-1}$ and the degradation half-life of NIS reduced from $11.5 \mathrm{~d}$ to $4.4 \mathrm{~d}$. Increased the initial concentrations of surfactants led to a little increase of the degradation half-life of LAS and NIS. The biodegradation of surfactants at $\mathrm{pH} 7$ was slightly easier than that of at $\mathrm{pH} 10$. The degradation of NIS and LAS could be inhibited by added glucose but be promoted by added $\mathrm{Na}_{2} \mathrm{HPO}_{4}$. Aeration could accelerate the degradation of LAS but had limited promotion on the degradation of NIS

Keywords: LAS; NIS; biodegradation;degradation kinetics; Lake Dianchi

表面活性剂(surfactant)与人们的生产生活息息相关,是一类品种多样、用途广泛的精细化工产品.主要 包括阳离子型(cationic)表面活性剂、阴离子型(anion)表面活性剂、非离子型(non-ion)表面活性剂和两性 离子(amphoteric)表面活性剂. 其中阴离子型和非离子型表面活性剂的产量约占总产量的 $90 \%^{[1]}$. 经过五

* 国家高技术研究发展计划(863)项目(2005AA60101005)和中国科学院重要方向项目(KZCX2-YW-426)联合资助. 2007-01-18 收稿; 2007-05-19 收修改稿. 王智, 男, 1983 年生, 硕士研究生; E-mail: wazh519@hotmail.com.

** 通讯作者; E-mail: bdxiao@ihb.ac.cn. 
十多年的发展，我国表面活性剂工业发展迅速. 根据塑料行业信息网提供的信息表明，2005年，我国表面 活性剂产量为 $301.8 \times 10^{4} \mathrm{t}$, 居世界第二位. 表面活性剂的大量使用，在造福人类的同时，也对水环境造成 污染, 我国环境标准中将表面活性剂列为第二类污染物质. 表面活性剂进人自然水体, 会造成水体起泡、 产生毒性, 且会降低水中的复氧速率和充氧程度, 使水质变坏, 影响水生生物的生存, 使水体自净受阻. 它还能乳化水体中其他的污染物质，增大污染物质的浓度,阻碍其它污染物质的降解，造成间接污染. 此 外, 表面活性剂具有抑制和杀死微生物的作用 ${ }^{[2]}$, 因此有关表面活性剂的降解愈来愈受到人们的重视 ${ }^{[3]}$. 滇池是我国著名的高原淡水湖泊, 位于昆明市的南端, 处在 200 多万人口城市的下水口, 整座城市的污水 都流往滇池. 近十几年来, 由于大量的生活污水和工业废水通过不同的河道流人滇池, 滇池北部水体已 经受到严重污染, 水质为劣 $\mathrm{V}$ 类. 其中, 大量的表面活性剂随污水流人滇池, 对滇池的水体产生不利影 响. 有关表面活性剂的生物降解, 各国学者都作了大量研究, 主要集中在研究表面活性剂的结构与生物 可降解性的关系 ${ }^{[4-7]}$ 以及利用驯化培养的菌株来降解表面活性剂废水中的高浓度的表面活性剂 ${ }^{[8-11]}$. 除 Quiroga ${ }^{[12]}$ 和Perales ${ }^{[13]}$ 对海水和河水中阴离子表面活性剂直链烷基苯磺酸钠(linear alkylbenzene sulfonates, LAS)的生物降解进行过动力学研究之外, 很少有人研究自然水体中表面活性剂在不同条件下的生物降解. 本文旨在研究一种常见阴离子表面活性剂直链烷基苯磺酸钠(LAS)和一种非离子表面活性剂(NIS)辛基酚 聚氧乙烯(10)梄(p-iso-octylphenol polyethoxylate, Triton X-100)在滇池水体中不同条件下的生物降解规律, 阐释表面活性剂在富营养化淡水湖泊中的环境特征, 从而为控制表面活性剂的废水排放提供科学依据.

\section{1 材料与方法}

\section{1 主要试剂及仪器}

试验中添加的主要物质: 阴离子表面活性剂直链烷基苯磺酸钠(LAS)、非离子表面活性剂(NIS) Triton X-100、葡萄糖、磷酸氢二钠均购自国药集团(A.R.级), 分析测试中其他所用主要药品: 三氯甲烷, 1, 2-二 氯乙烷，亚甲基蓝，苦味酸，氯化钡等药剂均为A.R.级.

$752 \mathrm{~N}$ 紫外可见分光光度计(上海精密科学仪器有限公司), SPX型智能生化培养箱(宁波东南仪器有限 公司), LXJ-II B低速大容量多管离心机(上海安亭科学仪器厂).

\section{2 试验方法}

本生物降解试验采用河水衰减法 ${ }^{[12-13]}$, 试验水样取自滇池北部福保湾外围水体的 $0-20 \mathrm{~cm}$ 表层,采样 时间为 2006 年 11 月 3 日, 采集时水体主要理化特征为: $\mathrm{T}=20.2^{\circ} \mathrm{C}, \mathrm{pH}=10.02, \mathrm{DO}=9.1 \mathrm{mg} / \mathrm{L}, \mathrm{TN}=5.8 \mathrm{mg} / \mathrm{L}$, $\mathrm{PO}_{4}{ }^{3-}=0.10 \mathrm{mg} / \mathrm{L}, \mathrm{Chl} . \mathrm{a}=0.187 \mathrm{mg} / \mathrm{L}, \mathrm{LAS}=75 \mu \mathrm{g} / \mathrm{L}, \mathrm{NIS}=151 \mu \mathrm{g} / \mathrm{L}$. 将水样充分混合均匀, 分装于 $2.5 \mathrm{~L}$ 细 口玻璃瓶中，每个瓶中加人 $2 \mathrm{~L}$ 水样，然后添加不同剂量表面活性剂，置于生化培养箱中于不同条件下避 光培养. 试验中设计的不同培养条件包括温度、 $\mathrm{pH}$ 值、表面活性剂初始浓度、添加营养物质及曝气(表 1 和表 2). 试验中之所以选择温度 $20^{\circ} \mathrm{C}, \mathrm{pH}=10$ 的培养条件, 主要是因为采集水样时, 滇池水温 $20.2^{\circ} \mathrm{C}, \mathrm{pH}$ 10.02 , 这样使模拟试验更接近实际. 滇池为典型的富营养化湖泊, 在试验中添加葡萄糖或磷酸氢二钠主 要是为了考察滇池水体微生物在有充足的碳源和磷源(葡萄糖或磷酸氢二钠为 $5 \mathrm{~g} / \mathrm{L}$ ) 时对 LAS 和 NIS 的降 解影响. 试验开始后每天测定 LAS 和 NIS 的含量, 连续测定 $30 \mathrm{~d}$. 每个试验条件均设置一平行对照, 试验 结果取平均值. 实验数据采用 Excel 和 OriginPro7.0 统计软件进行处理.

阴离子表面活性剂测定采用亚甲基蓝分光光度法(GB7497-87) ${ }^{[14]}$; 非离子表面活性剂的测定采用苦 味酸钡络合萃取比色法 ${ }^{[15]}$.

\section{2 结果与讨论}

\section{1 不同试验条件对表面活性剂降解的影响}

2.1.1 温度对 LAS 和 NIS 降解的影响 温度主要影响水体中的微生物的活性, 这种影响十分明显地表现在 二种表面活性剂降解曲线的差异上. 温度越高, 表面活性剂的降解速率越快, 其启动降解的滞后期也越 短.如 LAS 的半衰期在 $10 、 20 、 30^{\circ} \mathrm{C}$ 时分别为 $16.3 \mathrm{~d} 、 6.6 \mathrm{~d}$ 和 $3.2 \mathrm{~d}$, 其降解率达 $95 \%$ 时所需时间分别为 $28 \mathrm{~d}$ 、 $11 \mathrm{~d}$ 和 $5 \mathrm{~d}$. NIS 的降解类似于 LAS, 但其降解速率较慢. 从降解曲线可以清楚地看到, 在同一温度下, LAS 的 
降解速率明显快于 NIS, 如温度为 $10^{\circ} \mathrm{C}$ 时, 在 $30 \mathrm{~d}$ 内, NIS 的降解率仅为 $10 \%$, 而 LAS 的降解率达 $95 \%$ (图 1). 这表明, 在滇池水体中 LAS 的自然生物降解性要优于 NIS.

表 1 LAS 生物降解实验的培养条件

Tab.1 Different cultivation conditions of the biodegradation of LAS

\begin{tabular}{ccccccc}
\hline 序号 & $\begin{array}{c}\text { 表面活性剂 } \\
\text { 初始浓度 }(\mathrm{mg} / \mathrm{L})\end{array}$ & $\begin{array}{c}\text { 培养 } \\
\text { 温度 }\left({ }^{\circ} \mathrm{C}\right)\end{array}$ & $\begin{array}{c}\text { 初始 } \\
\mathrm{pH} \text { 值 }\end{array}$ & $\begin{array}{c}\text { 添加的葡萄糖 } \\
\text { 浓度 }(\mathrm{g} / \mathrm{L})\end{array}$ & $\begin{array}{c}\text { 添加磷酸氢二 } \\
\text { 钠浓度 }(\mathrm{g} / \mathrm{L})\end{array}$ & $\begin{array}{c}\text { 是否 } \\
\text { 曝气 }\end{array}$ \\
\hline 1 & 5.28 & 10 & 10 & 0 & 0 & 0 \\
2 & 5.28 & 20 & 10 & 0 & 0 & 否 \\
3 & 5.28 & 30 & 10 & 0 & 0 & 否 \\
4 & 5.28 & 20 & 7 & 0 & 0 & 否 \\
5 & 5.28 & 20 & 10 & 0 & 0 & 否 \\
6 & 5.28 & 20 & 12 & 0 & 0 & 否 \\
7 & 2.78 & 20 & 10 & 0 & 0 & 否 \\
8 & 5.28 & 20 & 10 & 0 & 0 & 否 \\
9 & 10.94 & 20 & 10 & 0 & 0 & 否 \\
10 & 5.28 & 20 & 10 & 0 & 5 & 否 \\
11 & 5.28 & 20 & 10 & 5 & 0 & 否 \\
12 & 5.28 & 20 & 10 & 0 & 0 & 否 \\
13 & 5.28 & 20 & 10 & 0 & 0 & 0 \\
14 & 5.28 & 20 & 10 & 0 & 0 & 0 \\
\hline
\end{tabular}

表 2 NIS 生物降解实验的培养条件

Tab.2 Different cultivation conditions of the biodegradation of NIS

\begin{tabular}{ccccccc}
\hline 序号 & $\begin{array}{c}\text { 表面活性剂 } \\
\text { 初始浓度 }(\mathrm{mg} / \mathrm{L})\end{array}$ & $\begin{array}{c}\text { 培养 } \\
\text { 温度 }\left({ }^{\circ} \mathrm{C}\right)\end{array}$ & $\begin{array}{c}\text { 初始 } \\
\mathrm{pH} \text { 值 }\end{array}$ & $\begin{array}{c}\text { 添加的葡萄糖 } \\
\text { 浓度 }(\mathrm{g} / \mathrm{L})\end{array}$ & $\begin{array}{c}\text { 添加磷酸氢二 } \\
\text { 钠浓度 }(\mathrm{g} / \mathrm{L})\end{array}$ & $\begin{array}{c}\text { 是否 } \\
\text { 曝气 }\end{array}$ \\
\hline 15 & 5.75 & 10 & 10 & 0 & 0 & 0 \\
16 & 5.75 & 20 & 10 & 0 & 0 & 否 \\
17 & 5.75 & 30 & 10 & 0 & 0 & 否 \\
18 & 5.75 & 20 & 7 & 0 & 0 & 否 \\
19 & 5.75 & 20 & 10 & 0 & 0 & 否 \\
20 & 5.75 & 20 & 12 & 0 & 0 & 否 \\
21 & 3.08 & 20 & 10 & 0 & 0 & 否 \\
22 & 5.75 & 20 & 10 & 0 & 0 & 否 \\
23 & 11.29 & 20 & 10 & 0 & 0 & 否 \\
24 & 5.75 & 20 & 10 & 0 & 0 & 否 \\
25 & 5.75 & 20 & 10 & 5 & 0 & 否 \\
26 & 5.75 & 20 & 10 & 0 & 0 & 否 \\
27 & 5.75 & 20 & 10 & 0 & 0 & 是 \\
28 & 5.75 & 20 & 10 & 0 & 0 & 0 \\
\hline
\end{tabular}

2.1.2 初始浓度对降解的影响 表面活性剂的初始浓度越高, 其生物降解的滞后期越长, 半衰期也越长(图 2). 如 LAS 初始浓度由 $2.78 \mathrm{mg} / \mathrm{L}$ 增至 $10.94 \mathrm{mg} / \mathrm{L}$ 时, 其降解滞后期由 $2 \mathrm{~d}$ 增至 $4 \mathrm{~d}$, 半衰期 $t_{1 / 2}$ 由 $6.1 \mathrm{~d}$ 增至 $7 \mathrm{~d}$; NIS 也呈现类似规律, 其初始浓度由 $3.08 \mathrm{mg} / \mathrm{L}$ 增至 $11.29 \mathrm{mg} / \mathrm{L}$ 时, 其降解滞后期由 $8 \mathrm{~d}$ 增至 $9 \mathrm{~d}$, 半衰 期 $t_{1 / 2}$ 由 $11.3 \mathrm{~d}$ 增至 $13.4 \mathrm{~d}$. 不同的初始浓度对表面活性剂降解的影响不同，主要原因可能是表面活性剂对 水体中的微生物有一定的毒性, 表面活性剂的初始浓度越高, 其对水体中微生物活性的抑制作用越大. 在本实验中选择表面活性剂最高初始浓度 $(\mathrm{LAS}=10.94 \mathrm{mg} / \mathrm{L}, \mathrm{NIS}=11.29 \mathrm{mg} / \mathrm{L}$ )时对滇池水体中的微生物活 性有比较明显的抑制作用，这与 Swisher ${ }^{[4]}$ 的结论 “表面活性剂对水体微生物活性产生明显抑制作用时的 
浓度高于 $10 \mathrm{mg} / \mathrm{L} ”$ 一致.

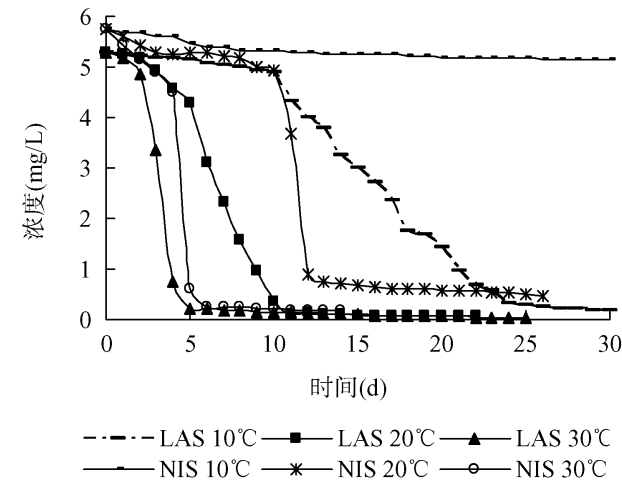

图 1 不同温度下 LAS 和 NIS 的降解曲线

Fig.1 The degradation curves of LAS and NIS under different temperatures

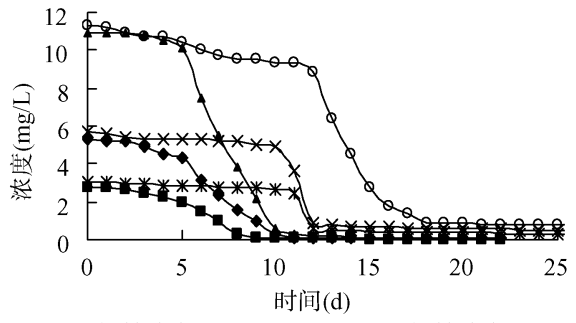

$\rightarrow$ LAS 初始浓度 $5.28 \mathrm{mg} / \mathrm{L} \rightarrow-\mathrm{LAS}$ 初始浓度 $2.78 \mathrm{mg} / \mathrm{L}$ —LAS 初始浓度 $10.94 \mathrm{mg} / \mathrm{L} \rightarrow$ NIS 初始浓度 $5.75 \mathrm{mg} / \mathrm{L}$ * N NIS 初始浓度 $3.08 \mathrm{mg} / \mathrm{L} \rightarrow-\mathrm{NIS}$ 初始浓度 $11.29 \mathrm{mg} / \mathrm{L}$

图 2 不同初始浓度 LAS 和 NIS 的降解曲线

Fig.2 The degradation curves of LAS and NIS at different initial concentrations

2.1.3 水体 $\mathrm{pH}$ 对降解的影响 一般来说, $\mathrm{pH}$ 值对水体微生物的活性有着直接影响. 其中 $\mathrm{pH}=10$ 为采样时 滇池水体的 $\mathrm{pH}$ 值. 不同 $\mathrm{pH}$ 条件下表面活性剂的降解规律也存在差异(图 3). 当 $\mathrm{pH}$ 为 7 和 10 时, LAS 的 降解曲线基本重合, 表明 $\mathrm{pH}$ 在 7-10 范围内, LAS 的生物降解无明显差异. 而当 $\mathrm{pH}$ 由 10 上升至 12 时, LAS 降解的滞后期由 $3 \mathrm{~d}$ 增至 $10 \mathrm{~d}$, 这可能是由于部分微生物不能适应 $\mathrm{pH}=12$ 的水环境, 导致启动生物降 解的时间向后延迟. 但是, 那些存活下来的微生物经过一段时间的适应和驯化后，一旦开始降解 LAS, 其降解可在 $7 \mathrm{~d}$ 内完成, 与 $\mathrm{pH}$ 为 7 或 10 时的降解完成时间差不多. 相比之下, $\mathrm{pH}$ 对 NIS 的降解影响较为 明显, $\mathrm{pH}$ 越大, NIS 的降解越缓慢, 降解滞后期也越长. 如 $\mathrm{pH}$ 为 $7 、 10$ 和 12 时, NIS 的降解滞后期为 $8 \mathrm{~d} 、 10 \mathrm{~d}$ 和 20d. 在同一 $\mathrm{pH}$ 时, LAS 的降解明显快于 NIS(图 3). 综合 LAS 和 NIS 在 $\mathrm{pH}$ 为 7、10 和 12 条件下的降 解规律, 可以认为, 在滇池自然水体中 $(\mathrm{pH}$ 一般为 8-9), $\mathrm{pH}$ 对表面活性剂的自然生物降解有一定影响, 但 是这种影响远不如温度的影响那样明显.

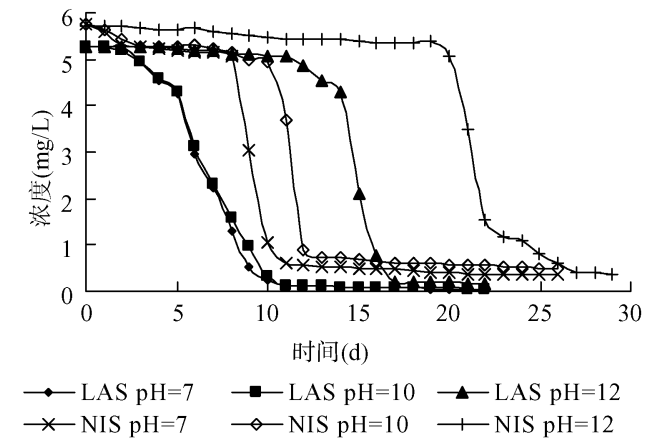

图 3 不同 $\mathrm{pH}$ 下 LAS 和 NIS 的降解曲线

Fig.3 The degradation curves of LAS and NIS at different $\mathrm{pH}$ values

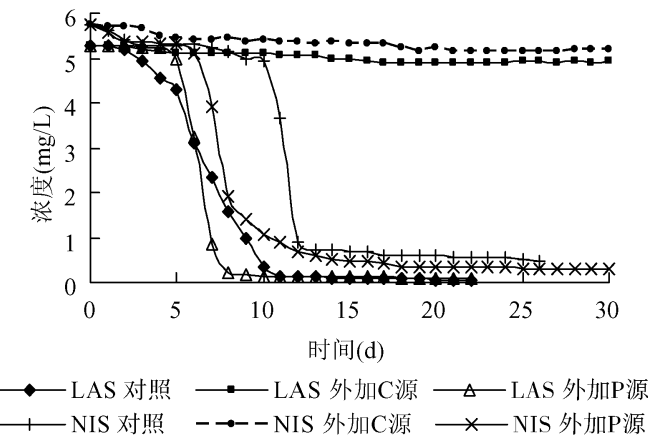

图 4 外加不同营养盐下 LAS 和 NIS 的降解曲线

Fig.4 The degradation curves of LAS and NIS at different nutrients added

2.1.4 模拟水体中添加营养物质对表面活性剂降解的影响 在本实验中, 探讨了培养温度为 $20^{\circ} \mathrm{C}$ 时, 在 模拟水体中添加营养物质对表面活性剂降解的影响. 添加葡萄糖或磷酸氢二钠的情况下 LAS 和 NIS 的降 解曲线可知(图 4), 当实验水体中添加足量的葡萄糖(5g/L)时, LAS 和 NIS 的降解均受到明显抑制, $30 \mathrm{~d}$ 的 降解率仅为 $10 \%$ 左右，这可能是由于葡萄糖比 LAS 和 NIS 能更好地被微生物优先利用，从而使 LAS 和 NIS 的降解受阻. 而添加足量的磷酸氢二钠 $(5 \mathrm{~g} / \mathrm{L})$ 时, LAS 的降解滞后期虽然比对照延迟 $2 \mathrm{~d}$, 但其降解速 度却较对照快，如在添加磷酸氢二钠的情况下, LAS 降解率达 $95 \%$ 所需时间为 $8 \mathrm{~d}$, 而对照组需要 $11 \mathrm{~d}$. 添 
加磷酸二氢钠对 NIS 的降解影响更为明显, 不仅缩短 了降解滞后期, 而且提高了 NIS 的降解速率. 虽然添 加磷酸氢二钠能促进水体中表面活性剂的自然生物降 解, 但由于磷是造成湖泊富营养化的主要生源要素, 属于需要优先控制的营养物质, 因此不能用添加磷等 营养物质的方法促进表面活性剂的降解.

2.1.5 曝气对降解的影响 曝气主要是增加水体中的 溶解氧(DO), 本实验研究了温度为 $20^{\circ} \mathrm{C}$ 时连续曝气对 水体中表面活性剂降解的影响. 曝气对 LAS 的降解具 有促进作用, 与对照相比, 曝气条件下 LAS 的降解速 率由 $0.73 \mathrm{~d}^{-1}$ 增至 $1.16 \mathrm{~d}^{-1}$, 半衰期由 $6.6 \mathrm{~d}$ 降至 $5.6 \mathrm{~d}$, 说 明 DO 的增加有利于水体中 LAS 的降解, 这与文献报

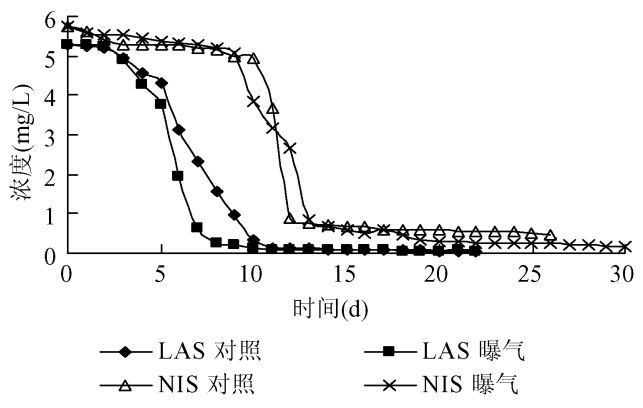

图 5 曝气条件下 LAS 和 NIS 的降解曲线 Fig. 5 The degradation curves of LAS and NIS under aeration condition 道 ${ }^{[16]}$ 的 LAS 在好氧和厌氧条件下降解差异很大的结论是一致的，但是曝气对 NIS 降解的促进作用并不明 显(图 5 和表 3). 在连续曝气的情况下, NIS 的降解速率 $\left(0.72 \mathrm{~d}^{-1}\right)$ 和半衰期 $(11.6 \mathrm{~d})$ 与对照 $\left(0.75 \mathrm{~d}^{-1}, 11.5 \mathrm{~d}\right)$ 差别 不大，这与文献报道 ${ }^{[17]}$ 的 NIS 中聚氧乙烯醚类表面活性剂的降解在好氧和厌氧下降解速度与程度基本相 同的结论也一致(图 5 和表 4).

表 3 LAS 生物降解半衰期及非线性拟合参数

Tab.3 Parameters of nonlinear curve fit and half-life of biodegradation of LAS

\begin{tabular}{cccccc}
\hline 序号 & $p(/ \mathrm{d})$ & $q(\mathrm{mg} / \mathrm{L})$ & $h(\mathrm{mg} / \mathrm{L})$ & $r^{2}$ & $t_{1 / 2}(\mathrm{~d})$ \\
\hline 1 & 0.31 & 0.06 & 5.31 & 0.997 & 16.3 \\
2 & 0.73 & 0.05 & 5.30 & 0.998 & 6.6 \\
3 & 2.10 & 0.10 & 5.28 & 0.999 & 3.2 \\
4 & 0.81 & 0.05 & 5.29 & 0.999 & 6.4 \\
5 & 0.73 & 0.05 & 5.30 & 0.998 & 6.6 \\
6 & 0.88 & -0.02 & 5.28 & 0.988 & 14.8 \\
7 & 0.84 & 0.03 & 2.79 & 0.997 & 6.1 \\
8 & 0.73 & 0.05 & 5.30 & 0.998 & 6.6 \\
9 & 0.90 & 0.10 & 10.95 & 0.997 & 7.0 \\
10 & 0.73 & 0.05 & 5.30 & 0.998 & 6.6 \\
11 & - & - & - & - & $>30$ \\
12 & 0.97 & -0.02 & 5.28 & 0.978 & 6.2 \\
13 & 0.73 & 0.05 & 5.30 & 0.998 & 6.6 \\
14 & 1.16 & 0.06 & 5.28 & 0.997 & 5.6 \\
\hline
\end{tabular}

\section{2 表面活性剂的生物降解动力学}

各培养条件下表面活性剂都有一定程度的降解，除个别培养条件外，表面活性剂在一定时间内都几 乎可以完全降解，LAS 的降解率达 $95 \%$ 以上, NIS 的降解率达 $92 \%$ 以，其降解曲线随时间呈“S”形变化 (图 1-图 5). 一般来说, LAS 和 NIS 的降解过程与水体中微生物的生长代谢密切相关, 这种过程可用下式 来表述 ${ }^{[12-13]}$ :

$$
-\frac{\mathrm{d} S}{\mathrm{~d} t}=K_{2} \cdot S^{2}+K_{1} \cdot S+K_{0}
$$

解(1)式, 得到表面活性剂浓度与时间的关系方程为:

$$
S=\frac{h\left(S_{0}-q\right)-q\left(S_{0}-h\right) \exp (p t)}{\left(S_{0}-q\right)-\left(S_{0}-h\right) \exp (p t)}
$$


其中

$$
p=\sqrt{K_{1}^{2}-4 K_{2} K_{0}} ; q=\frac{\left(-K_{1}+p\right)}{2 K_{2}} ; h=\frac{\left(-K_{1}-p\right)}{2 K_{2}} .
$$

式中, $K_{2}$ 为 $S^{2}$ 的系数, $K_{1}$ 为 $S$ 的系数, $K_{0}$ 为常数, $t$ 为降解时间 (d), $S$ 为表面活性剂在 $t$ 时的浓度 $(\mathrm{mg} / \mathrm{L}), S_{0}$ 为表面活性剂的初始浓度 $(\mathrm{mg} / \mathrm{L}), p$ 为降解作用发生后微生物对表面活性剂的降解速率 $\left(\mathrm{d}^{-1}\right), q$ 为微生物不 能利用的表面活性剂的浓度 $(\mathrm{mg} / \mathrm{L}), h$ 为培养介质中微生物能利用的最大表面活性剂浓度 $(\mathrm{mg} / \mathrm{L})$.

利用上述实验数据进行非线性拟合表明，在不同条件下(除序号 $11 、 15$ 和 25 外), 表面活性剂的生物 降解过程在 $95 \%$ 置信水平上符合式(2), 通过拟合方程计算出表面活性剂降解半衰期 $t_{1 / 2}$ (d) (表 3, 表 4).

表 4 NIS 生物降解半衰期及非线性拟合参数

Tab.4 Parameters of nonlinear curve fit and half-life of biodegradation of NIS

\begin{tabular}{cccccc}
\hline 序号 & $p(/ \mathrm{d})$ & $q(\mathrm{mg} / \mathrm{L})$ & $h(\mathrm{mg} / \mathrm{L})$ & $r^{2}$ & $t_{1 / 2}(\mathrm{~d})$ \\
\hline 15 & - & - & - & - & $>30$ \\
16 & 0.75 & 0.44 & 5.75 & 0.964 & 11.5 \\
17 & 2.07 & 0.04 & 5.75 & 0.978 & 4.4 \\
18 & 1.36 & 0.44 & 5.75 & 0.981 & 9.1 \\
19 & 0.75 & 0.44 & 5.75 & 0.964 & 11.5 \\
20 & 0.67 & 0.03 & 5.73 & 0.976 & 21.3 \\
21 & 0.75 & 0.30 & 3.08 & 0.956 & 11.3 \\
22 & 0.75 & 0.44 & 5.75 & 0.964 & 11.5 \\
23 & 0.53 & 0.37 & 11.30 & 0.978 & 13.4 \\
24 & 0.75 & 0.44 & 5.75 & 0.964 & 11.5 \\
25 & - & - & - & - & $>30$ \\
26 & 0.99 & 0.46 & 5.75 & 0.991 & 7.5 \\
27 & 0.75 & 0.44 & 5.75 & 0.964 & 11.5 \\
28 & 0.72 & 0.28 & 5.75 & 0.993 & 11.6 \\
\hline
\end{tabular}

\section{3 结论}

(1)在滇池自然水体中, 温度、表面活性剂的初始浓度、 $\mathrm{pH}$ 值、添加营养物质(葡萄糖或磷酸氢二钠) 和有无曝气都对表面活性剂的降解有一定的影响. 温度是滇池水体中表面活性剂降解的主要影响因素,在 $10-30^{\circ} \mathrm{C}$ 时, 温度越高, 降解速率越快, 降解半衰期越短; 表面活性剂的初始浓度越高, 其生物降解的滞后 期越长, 半衰期也越长; 滇池水体 $\mathrm{pH}$ 值在 7-10 时, 水体中的表面活性剂都能得到很好的降解, $\mathrm{pH}$ 为 7 时的降解速率略高于 $\mathrm{pH}$ 为 10 时的降解速率; 在表面活性剂和葡萄糖碳源共存的情况下, 滇池水体中微 生物优先利用碳源葡萄糖, 30d 内表面活性剂降解率不足 $10 \%$, 而添加磷源磷酸氢二钠对其降解有一定的 促进作用; 曝气能促进 LAS 的降解, 但是对 NIS 降解的促进作用并不明显.

(2)在滇池的自然水体中, LAS 和 NIS 都能自然生物降解, 实验 $30 \mathrm{~d}$ 后, LAS 的降解率达 $95 \%$ 以上, NIS 的降 解率达 $92 \%$ 以上(NIS 在 $10^{\circ} \mathrm{C}$ 时除外, $30 \mathrm{~d}$ 内, 降解率仅为 $10 \%$ ), 降解遵从二级动力模型 $-\mathrm{d} S / \mathrm{d} t=K_{2} \cdot \mathrm{S}^{2}+K_{I} \cdot \mathrm{S}+K_{0}, r^{2}$ 值为 0.956-0.999, LAS 的降解速率 $p$ 值为 $0.31-2.10 \mathrm{~d}^{-1}$, 降解的半衰期 $t_{1 / 2}$ 为 3.2-16.3d; NIS 的降解速率 $p$ 值为 $0.53-2.07 \mathrm{~d}^{-1}$, 降解半衰期 $t_{1 / 2}$ 为 $4.4-21.3 \mathrm{~d}$.

(3)在滇池水体中, LAS 的自然生物降解性能优于 NIS(Triton X-100)的自然生物降解性能.

\section{4 参考文献}

[1] 魏福祥, 郝莉莉, 王金梅. 表面活性剂对环境的污染及检测研究进展. 河北工业科技, 2006, 23(1): 57-60.

[2] 王 晗, 张清军, 白长琦等. 含表面活性剂稠油污水生化降解试验研究. 河南石油, 2004, 18(6)(增刊): 75-78. 
[3] 杨丽娟. 表面活性剂生物降解和光降解技术研究进展. 精细化工, 2002, 19(8)(增刊): 113-115.

[4] Swisher RD. Surfactant biodegradation. New York : Marcel Dekker Inc, 1987: 424

[5] Birch R. Biodegradation of nonionic surfactant. J Am Oil Chem Soc, 1984, 61: 340-343.

[6] Sturm RN. Biodegradation of nonionic surfactant: screening test for predicting rate and ultimate biodegradation. $J$ Am Oil Chem Soc, 1973, 50: 159-167.

[7] Baker Irene JA. Sugar fatty acid surfactant: structure and ultimate aerobic biodegradation. Journal of Surfactants and Detergents, 2000, 3(1): 1-32.

[8] 应启锋, 肖昌松, 纪树兰. 直链烷基苯磺酸钠(LAS)降解菌的篮选及其降解特性的初步研究. 微生物学通报, 2002, 29(1): 1-5.

[9] 张蔚文, 张 灼. 降解直链烷基苯磺酸钠 LAS 菌株的笁选及其降解质粒的研究. 环境科学与技术, 1991, (3): 2-6.

[10] 刘秀荣, 吕晓猛, 纪树兰. 微生物降解直链十二烷基苯磺酸钠的研究. 北京工业大学学报, 1995, 21(4): 103-108.

[11] 冯 树, 周樱桥, 张忠泽. 微生物混合培养及其应用. 微生物学通报, 2001, 28(3): 92-95.

[12] Quiroga JM, Perales JA, Romero LI et al. Biodegradation Kinetics of surfactants in seawater. Chemosphere, 1999, 39(11): 1957-1969.

[13] Perales JA, Manzano MA, Sales D et al. Biodegradation kinetics of LAS in river water. International Biodeterioration \& Biodegradation, 1999, 43: 155-160.

[14] 吴季松, 刘雅鸣. 水利技术标准汇编: 水资源水环境卷·分析方法. 北京: 中国水利水电出版社, 2002: 462-468.

[15] 钱锡兴, 王敬名, 胡振元. 水中痕量非离子表面活性剂的测定. 分析化学, 1987, 15(2): 60-61.

[16] 官景渠, 李济生. 表面活性剂在环境中的生物降解. 环境科学, 1993, 15(2): 81-85. 